\title{
Human cytomegalovirus RL13 protein interacts with host NUDT14 protein affecting viral DNA replication
}

\author{
GUILI WANG ${ }^{1,2}$, GAOWEI REN $^{1}$, XIN CUI $^{1}$, ZHITAO LU $^{1}$, YANPING MA ${ }^{1}$, YING QI $^{1}$, \\ YUJING HUANG ${ }^{1}$, ZHONGYANG LIU ${ }^{1}$, ZHENGRONG SUN ${ }^{1}$ and QIANG RUAN ${ }^{1}$ \\ ${ }^{1}$ Virus Laboratory, The Affiliated Shengjing Hospital, China Medical University, Shenyang, Liaoning 110004; \\ ${ }^{2}$ Department of Pediatrics, The Third Affiliated Hospital of Liaoning Medical College, Jinzhou, Liaoning 121001, P.R. China
}

Received March 6, 2015; Accepted December 18, 2015

DOI: $10.3892 / \mathrm{mmr} .2016 .4778$

\begin{abstract}
The interaction between the host and human cytomegalovirus (HCMV) is important in determining the outcome of a viral infection. The HCMV RL13 gene product exerts independent, inhibitory effects on viral growth in fibroblasts and epithelial cells. At present, there are few reports on the interactions between the HCMV RL13 protein and human host proteins. The present study provided direct evidence for the specific interaction between HCMV RL13 and host nucleoside diphosphate linked moiety $\mathrm{X}$ (nudix)-type motif 14 (NUDT14), a UDP-glucose pyrophosphatase, using two-hybrid screening, an in vitro glutathione S-transferase pull-down assay, and co-immunoprecipitation in human embryonic kidney HEK293 cells. Additionally, the RL13 protein was shown to co-localize with the NUDT14 protein in the HEK293 cell membrane and cytoplasm, demonstrated using fluorescence confocal microscopy. Decreasing the expression level of NUDT14 via NUDT14-specific small interfering RNAs increased the number of viral DNA copies in the HCMV-infected cells. However, the overexpression of NUDT14 in a stably expressing cell line did not affect viral DNA levels significantly in the HCMV infected cells. Based on the known functions of NUDT14, the results of the present study suggested that the interaction between the RL13 protein and NUDT14 protein may be involved in HCMV DNA replication, and that NUDT14 may offer potential in the modulation of viral infection.
\end{abstract}

Correspondence to: Professor Zhengrong Sun or Professor Qiang Ruan, Virus Laboratory, The Affiliated Shengjing Hospital, China Medical University, 36 Sanhao Street, Shenyang, Liaoning 110004, P.R. China

E-mail: sunzr@sj-hospital.org

E-mail: ruanq@sj-hospital.org

Key words: human cytomegalovirus, RL13, NUDT14, small interfering RNA, human cytomegalovirus replication

\section{Introduction}

One of the most ubiquitous and important pathogens worldwide is human cytomegalovirus (HCMV) (1-4). HCMV can cause serious diseases in specific groups of individuals, including those with compromised immunity and neonates. The viral infection may lead to congenital malformations in newborns and multi-organ involvement in patients with reduced immunity, with a wide range of cell tropism in HCMV infection (5-7).

The HCMV genome is $\sim 230 \mathrm{~kb}$ long and comprises 165 open reading frames in clinical strains (8). The HCMV RL13 open reading frame is commonly 909 bp in length in Han clinical strains, one of the RL11 gene families reported in our previous study (9). HCMV RL13 encodes a virion envelope glycoprotein, which locates spatially on the outer surface of the viral envelope $(10,11)$. The inhibitory effects of RL13 have been shown with clinical isolates, as well as with the bacterial artificial chromosome-cloned genome in fibroblasts and epithelial cells $(10,11)$. A previous study reported that RL13-encoded protein can bind human IgG and may contribute to HCMV immune evasion (12). Thus, more detailed investigations are required to determine the mechanism by which RL13 potentially targets unknown host proteins involved in viral replication.

The present study aimed to characterize the interaction and co-localization of RLI3 and nucleoside diphosphate linked moiety X (nudix)-type motif 14 (NUDT14) and provide evidence that this interaction may be involved in HCMV DNA replication. This may elucidate the molecular mechanisms underlying HCMV diseases and aid in indicating an appropriate target for effective therapeutic agents.

\section{Materials and methods}

Cells and virus. Astrocytoma U373MG cells were provided by Professor Songya Lv from State Key Laboratory of Virology, College of Life Sciences, Wuhan University (Wuhan, China). Human embryonic lung fibroblast MRC-5 cells (Shanghai Institute of Biochemistry and Cell Biology, Shanghai, China), human embryonic kidney HEK293 cells (Shanghai Institute of Cell Biology and Biochemistry) and the astrocytoma U373MG cells were cultured in Dulbecco's modified Eagle's medium 
(DMEM; GE Healthcare Life Sciences, Logan, UT, USA), which was mixed with $10 \%$ fetal bovine serum (GE Healthcare Life Sciences) at $37^{\circ} \mathrm{C}$ and $5 \% \mathrm{CO}_{2}$. A low-passage clinical isolate Han strain, which was isolated from a urine sample from a congenitally HCMV-infected infant at the Department of Pediatrics and maintained in the Virus Laboratory of The Affiliated Shengjing Hospital, China Medical University (Shenyang, China) was propagated in MRC-5 cells.

Yeast two-hybrid screening. The RL13 gene coding sequence was amplified by polymerase chain reaction using HCMV Han strain DNA (Genbank no. KJ426589.1; www.ncbi.nlm.nih. gov) as a template, and the following primers, obtained from Invitrogen (Thermo Fisher Scientific, Inc., Waltham, MA, USA): Forward 5'-CCGGGCCATGGAGGCCAATAACAC GTGCTCC-3' and reverse 5'-CGCGGATCCGGTACCTTA GGTTTTAGTCCA-3'. The PCR amplification was performed in a volume of $50 \mu \mathrm{l}$ containing $3.5 \mu \mathrm{l}$ DNA, $1 \mu \mathrm{l}$ each primer, $5 \mu 1$ 10X TaqBuffer (with $\mathrm{MgCl}_{2}$ ), $2.5 \mu \mathrm{l}$ dNTP mix (10 Mm), 1 unit Taq DNA polymerase (1 U/ $/ \mu 1$; Takara Biotechnology Co., Ltd., Dalian, China) and water up to $50 \mu \mathrm{l}$. The reaction was performed in a Bio-Rad MyCycler Thermal Cycler (Bio-Rad Laboratories, Inc., Hercules, CA, USA) and the reaction conditions were as follows: $95^{\circ} \mathrm{C}$ for $5 \mathrm{~min} ; 30$ cycles of $95^{\circ} \mathrm{C}$ for $45 \mathrm{sec}, 50^{\circ} \mathrm{C}$ for $45 \mathrm{sec}$ and $72^{\circ} \mathrm{C}$ for $1 \mathrm{~min}$; followed by a final elongation at $72^{\circ} \mathrm{C}$ for $10 \mathrm{~min}$. The PCR products were electrophoresed in $1.2 \%$ agarose gel containing ethidium bromide (Sigma-Aldrich, St. Louis, MO, USA) for visualization. The PCR products and the plasmids of pGBKT7 vector (Clontech Laboratories, Inc., Mountain View, CA, USA) were purified by wizard Genomic DNA Purification Kit (Promega Corporation, Madison, WI, USA) in accordance with the manufacturer's protocol. To construct the pGBKT7-RL13 plasmids, enzyme digestion was performed by BamHI (Takara Biotechnology Co., Ltd.) at $30^{\circ} \mathrm{C}$ for $2 \mathrm{~h}$ and SfiI (Takara Biotechnology Co., Ltd.) at $50^{\circ} \mathrm{C}$ for $1 \mathrm{~h}$. Following digestion, the products were purified by wizard Genomic DNA Purification Kit (Promega Corporation). Subsequently, the RL13 sequence was inserted into the pGBKT7 vector (Clontech Laboratories, Inc) between the SfiI and BamHI sites downstream of the c-Myc coding sequence by T4 DNA ligase (Takara Biotechnology Co., Ltd.) and T4 DNA ligase buffer (Takara Biotechnology Co., Ltd.) at $16^{\circ} \mathrm{C}$ overnight, resulting in pGBKT7-RL13 plasmids. The inserted sequence of pGBKT7-RL13 was confirmed by sequencing (Invitrogen; Thermo Fisher Scientific, Inc.). Yeast two-hybrid experiments were performed in accordance with the manufacturer's protocol (Matchmaker GAL4 Two-Hybrid System 3; Clontech Laboratories, Inc.). The human fetus brain cDNA library was provided by Professor Gengfu Xiao from the State Key Laboratory of Virology, College of Life Sciences, Wuhan University, which was cloned into pACT2 (Clontech Laboratories, Inc.), and the pGBKT7-RL13 were co-transformed into the Saccharomyces cerevisiae strain, AH109 (Clontech Laboratories, Inc.) by electroporation (Bio-Rad Laboratories, Inc.) according to the protocol provided by the manufacturer. Positive colonies were screened on synthetic dropout medium (Clontech Laboratories, Inc.) in the absence of tryptophan, leucine, adenine and histidine, and were detected using a chromogenic reaction of $x-\alpha-G a l$ (Clontech Laboratories, Inc.) for $\alpha$-galactosidase activity (13).
The plasmids containing sequences encoding the interaction candidates of RL13, termed pACT2-cDNA, were extracted and rescued via electrotransformation into competent Escherichia coli DH5a (Clontech Laboratories, Inc.). Each pACT2-cDNA plasmid was co-transformed into yeast with either the pGBKT7-RL13 vector or the pGBKT7 empty vector. The transformed cells were selected on QDO plates and underwent assessment for $\beta$-galactosidase activity. Human gene sequences from blue yeast colonies were sequenced (Invitrogen; Thermo Fisher Scientific, Inc.), and analyzed using the BLAST database (http://www.ncbi.nlm.gov/blast).

Glutathione S-transferase (GST) pull-down experiments. The coding sequence of NUDT14, one of the candidate proteins interacting with RL13 protein, was obtained from a NUDT14-containing cDNA clone (pACT2-NUDT14), and was GST-tagged by cloning into the pGEX-4T-2 vector (Clontech Laboratories, Inc.) between the EcoRI and $\mathrm{XhoI}$ sites, yielding pGEX-4T2-NUDT14.

A GST-pull-down experiment was performed, according to the manufacturer's protocol (MagneGST ${ }^{\mathrm{TM}}$ Pull-Down System; Promega Corporation). The c-Myc-labeled RL13 (c-Myc-RL13) was expressed from pGBKT7-RL13 in a quick-coupled transcription/translation reaction (TNT) T7 Quick Reaction (14). GST-labeled NUDT14 (GST-NUDT14) was expressed in BL21 (DE3) cells (Tiangen Biotech Co., Ltd., Beijing, China) transfected with pGEX-4T2-NUDT14 and induced with isopropyl $\beta$-D-1-thiogalactopyranoside (IPTG; Clontech Laboratories, Inc.). Subsequently, the MagneGST particles were incubated with the reaction products for $30 \mathrm{~min}$ at room temperature. As a bait protein, $20 \mu 1$ of GST-NUDT14 was incubated with $80 \mu \mathrm{lc}$-Myc-RL13 for $1.5 \mathrm{~h}$ on a rotating platform at room temperature. Following washing with a GST binding/wash buffer (MagneGST ${ }^{\text {TM }}$ Pull-Down System) three times, the bound proteins on the MagneGST particles were eluted using elution buffer and solubilized in 2X SDS sample buffer (Beyotime Institute of Biotechnology, Haimen, China). The protein concentration was measured by ultraviolet spectrophotometry as previously described (15). The binding proteins $(20 \mathrm{mg})$ were loaded in each lane of $12 \%$ polyacrylamide gels (SDS-PAGE; Sigma-Aldrich) and the proteins were separated by gel electrophoresis at a constant voltage of $100 \mathrm{~V}$. The separated proteins were transferred electrically onto nitrocellulose membranes (Sigma-Aldrich), and then blocked with 5\% bovine serum albumin (BSA; Thermo Fisher Scientific, Inc.) for $2 \mathrm{~h}$. Western blot analyses were performed using monoclonal mouse anti-human antibodies against c-Myc (1:1,000; Thermo Fisher Scientific, Inc.; cat. no. AHO0052) or polyclonal goat anti-human antibodies against GST monoclonal antibodies (1:1,000; Thermo Fisher Scientific, Inc.; cat. no. PA5-18394) incubated overnight at $4^{\circ} \mathrm{C}$ and corresponding goat anti-mouse peroxidase-conjugated secondary antibodies (1:2,000; Beyotime Institute of Biotechnology; cat. no. A0216) and rabbit anti-goat peroxidase-conjugated immunoglobulin $\mathrm{G}$ (IgG) secondary antibodies (1:2,000; Absin Bioscience, Inc., Shanghai, China; cat. no. abs20005) incubated for $2 \mathrm{~h}$ at room temperature. The membranes were then reacted with the chemiluminescent substrate, which was provided in the Western Chemiluminescent Substrate kit (Pierce; Thermo Fisher Scientific, Inc.). Signals were determined using a Molecular 
Imager ChemiDoc XRS System (Bio-Rad Laboratories, Inc.). In the GST-pull-down assay, parallel experiments were performed in controls containing GST- or myc-tagged protein.

Co-immunoprecipitation analysis. The coding sequence of RL13 in pGBKT7-RL13 was obtained and inserted into the pCMV-myc (Clontech Laboratories, Inc.) between the SfiI and NotI sites, and designated as pCMV-myc-RL13. The coding sequence of NUDT14 in pGEX-4T2-NUDT14 was obtained and cloned into the EcoRI and XhoI sites of pCMV-HA (Clontech Laboratories, Inc.), and designated as pCMV-HA-NUDT14. The constructs were confirmed by gene sequencing (Invitrogen; Thermo Fisher Scientific, Inc.) The plasmids of pCMV-myc-RL13 and pCMV-HA-NUDT14 were transiently co-transfected into HEK293 cells using Lipofectamine 2000, according to the manufacturer's protocol (Invitrogen; Thermo Fisher Scientific, Inc.). The cell lysates were harvested with lysis buffer $\left(\right.$ M-PER $^{\mathrm{TM}}$ Mammalian Protein Extraction Reagent; Thermo Fisher Scientific, Inc.) supplemented with the protease inhibitors contained within the Protein G Immunoprecipitation kit (Promega Corporation). The protein concentration was determined using ultraviolet spectrophotometry (15). The co-immunoprecipitation analysis was performed, according to the manufacturer's protocols of the ProFound Mammalian c-myc Tag immunoprecipitation (IP)/Co-IP and hemagglutinin (HA) Tag IP/Co-IP kits (Pierce; Thermo Fisher Scientific, Inc.). The binding proteins (20 mg) were loaded in each lane of $12 \%$ polyacrylamide gels (SDS-PAGE) and the proteins were separated by gel electrophoresis. The separated proteins were electrotransferred onto nitrocellulose membranes (Sigma-Aldrich, St. Louis, MO, USA), and then blocked with 5\% BSA (Thermo Fisher Scientific, Inc.) for $2 \mathrm{~h}$. The expression levels of myc-RL13 and HA-NUDT14 were detected using Western blotting with mouse anti-human against myc or rabbit anti-human polyclonal antibodies against HA (1:100; Thermo Fisher Scientific, Inc., cat. no. 71-5500) incubated overnight at $4^{\circ} \mathrm{C}$ and corresponding peroxidase-conjugated goat anti-mouse and goat anti-rabbit (1:2,000; Beyotime Institute of Biotechnology; cat. no. A0208) IgG secondary antibodies incubated at room temperature for $2 \mathrm{~h}$. Imaging was performed using ChemiDoc ${ }^{\mathrm{TM}} \mathrm{XRS}+$ (Bio-Rad Laboratories, Inc.) and density analysis was conducted with Image J version 10.2 (National Institutes of Health, Bethesda, MD, USA). Parallel co-immunoprecipitation experiments were performed with specific tagged proteins containing myc or HA.

Cellular localization assay. To establish expression of the RL13 fusion protein with a green fluorescent protein (EGFP) tag, the RL13 coding region was amplified from the HCMV Han strain using the following primers: Forward 5'-CGCTCGAGCCAA TAACACGTGCTCC-3' and reverse 5'-CGCGGATCCTTA GGTTTTAGTCCA-3'. Subsequently, the product was inserted into the pEGFP-C1 (Clontech Laboratories, Inc.) via the XhoI and BamHI sites, yielding pEGFP-C1-RL13. Similarly, the coding sequence of NUDT14 in the NUDT14-containing cDNA clone was fused to the Discosoma sp. red fluorescent protein (DsRed) tag via amplification using PCR with the following primers: Forward 5'-CGGAATTCCGTGTTGGTG AAGCAG-3' and reverse 5'-CGCGGATCCGGAGCTATG
CAAGCC-3', and cloned into the pDsRed-C1 (Clontech) via the EcoRI and BamHI sites, yielding pDsRed-C1-NUDT14. The constructs were confirmed by gene sequencing (Invitrogen; Thermo Fisher Scientific, Inc.).

The HEK293 cells $\left(5 \times 10^{6}\right)$ were seeded into a $35 \mathrm{~mm}$ confocal microscope dish (Nest Biotechnology Co., Ltd, Jiangsu, China) $24 \mathrm{~h}$ prior to transfection. At $75 \%$ confluence, the cells were transfected with either $4 \mu \mathrm{g}$ pEGFP-C1-RL13, $4 \mu \mathrm{g}$ pDsRed-C1-Nudt14, or a mixture of $2 \mu \mathrm{g}$ pEGFP-C1-RL13 and $2 \mu \mathrm{g}$ pDsRed-C1-NUDT14 using Lipofectamine 2000 (Invitrogen; Thermo Fisher Scientific, Inc.), according to the manufacturer's protocol. At $48 \mathrm{~h}$ post-transfection, the cells were subjected to DAPI staining (Invitrogen; Thermo Fisher Scientific, Inc.), and the expression levels of EGFP-RL13 and DsRed-NUDT14 were detected using a laser scanning confocal microscope (Nikon Eclipse C1 Plus; Nikon, Tokyo, Japan) with 488 and $543 \mathrm{~nm}$ excitation beams, which corresponded to the EGFP-RL13 and DsRed-NUDT14 proteins, respectively.

Stable expression of NUDT14 and transfection of NUDT14-specific small interfering RNA (siRNA) into cells. To generate a U373MG cell line, which stably expressed NUDT14 (U373-S), the pDsRed-C1-NUDT14 vector and empty vector, pDsRed-C1, were transfected into U373 cells using Lipofectamine $^{\circledR}$ LTX with Plus ${ }^{\mathrm{TM}}$ Reagent, according to the manufacturer's protocol (Invitrogen; Thermo Fisher Scientific, Inc.), yielding U373-S, and its control cell line, U373-C, respectively. At $48 \mathrm{~h}$ post-transfection, neomycin (Thermo Fisher Scientific, Inc.) was at a final concentration of $600 \mu \mathrm{g} / \mathrm{ml}$ following mixing with culture medium. Neomycin-resistant cells were screened with neomycin at $\sim 2$ weeks $(16,17)$. Protein extraction and quantification were performed as described previously. Western blot analysis was used to analyze the expression levels of NUDT14 in the selected cell clones, using rabbit anti-human polyclonal antibodies against NUDT14 (1:1,000; Abcam, Cambridge, MA, USA; cat. no. ab139656) and goat anti-rabbit IgG antibody conjugated with horseradish peroxidase. Equal quantities of sample were detected using western blotting, with antibodies against NUDT14. The antibody was characterized and specific bands for the NUDT14 protein were detected. As an internal control, the expression level of cellular glyceraldehyde-3-phosphate dehydrogenase (GAPDH) was analyzed by western blotting using mouse anti-human monoclonal antibody against GAPDH $(1: 1,000$; Abcam; cat. no. ab9482) and goat anti-mouse antibody conjugated with horseradish peroxidase. The primary antibodies were incubated overnight at $4^{\circ} \mathrm{C}$ and the secondary antibodies were incubated for $2 \mathrm{~h}$ at room temperature.

The cells $\left(n=1 \times 10^{5}\right)$ were cultured and transfected with Silencer ${ }^{\circledR}$ Select Pre-Designed NUDT14 siRNAs (Ambion; Thermo Fisher Scientific, Inc.) and control siRNA (C-siRNA) (Ambion; Thermo Fisher Scientific, Inc.), respectively, using Lipofectamine RNAiMAX Reagent, according to the manufacturer's protocol (Invitrogen; Thermo Fisher Scientific, Inc.). The antisense sequences of the NUDT14-specific siRNAs were: 5'-UUGAAUAAGAGAACGGUCAcg-3' (S-siRNA-1) and 5'-AAAGAUGACGCCGAGGGUCtt-3' (S-siRNA-2). In each well, $0.5 \mu 110 \mathrm{nM}$ siRNA and $3 \mu 1$ Lipofectamine (Invitrogen, Carlsbad, CA, USA) were diluted separately in $100 \mu \mathrm{l}$ Opti-MEM (Invitrogen; Thermo Fisher Scientific, Inc.). 
Table I. Homologous genes and homology were analyzed by comparing the gene sequences of positive clones with the human genome from Genebank.

\begin{tabular}{llr} 
Sequence number & \multicolumn{1}{c}{ Homologous genes } & Homology \\
\hline I & $\begin{array}{l}\text { Homo sapiens ankyrin repeat and GTPase domain Arf GTPase activating } \\
\text { protein 11 (AGAP11) }\end{array}$ & 100 \\
II & Homo sapiens ligase IV, DNA, ATP-dependent (LIG4) & 99 \\
III & Homo sapiens tripartite motif containing 2 (TRIM2) & 99 \\
IV & Homo sapiens tweety family member 3 (TTYH3) & 99 \\
V & Homo sapiens Sad1 and UNC84 domain containing 2 (SUN2) & 99 \\
VI & Homo sapiens aspartate beta-hydroxylase domain containing 1(ASPHD1) & 100 \\
VII & Homo sapiens G elongation factor, mitochondrial 2 (GFM2) & 98 \\
VIII & Homo sapiens ring finger protein 19A, RBR E3 ubiquitin protein ligase (RNF19A) & 94 \\
IX & Homo sapiens leptin receptor overlapping transcript-like 1(LEPROTL1) & 99 \\
X & Homo sapiens nucleoside diphosphate linked moiety X (nudix)-type motif 14 (NUDT14) & 100
\end{tabular}

Gene sequencing results of positive colonies, which were screened in a yeast two-hybrid experiment, were analyzed using the BLAST network server at the national center for biotechnology information ( http://www.ncbi.nlm.gov/blast). A total of 10 homologous genes and the corresponding homology were obtained on comparison with the human genome in the Genebank. Homologous genes were numbered I-X.

Following incubation for $5 \mathrm{~min}$ at room temperature, the two solutions were mixed. After 20 min, the mixture was added to the cells. At $10 \mathrm{~h}$ post-transfection, the medium containing the siRNA was discarded, and the transfection was repeated once to increase the transfection efficiency and interference efficiency. At $24 \mathrm{~h}$ following the secondary transfection, the cells were inoculated with the HCMV Han strain at a multiplicity of infection of one. Total DNAs and proteins were extracted from the infected cells $72 \mathrm{~h}$ following infection, and the levels of NUDT14 in the infected cells were determined using Western blot analysis.

Quantitative PCR ( $q P C R$ ) analysis. In brief, DNA was extracted from the HCMV-infected cells using a TIANamp Genomic DNA kit (Tiangen Biotech Co., Ltd.), according to the manufacturer's protocol. The viral DNAs were amplified and quantified using HCMV UL83-specific primers (forward 5'-GTCAGCGTTCGTGTTTCCCA-3' and reverse 5'-GGGACACAACACCGTAAAGC-3') and a SYBR Green PCR Master Mix kit (Applied Biosystems; Thermo Fisher Scientific, Inc.) on an ABI Prism 7300 Sequence Detection System (Applied Biosystems; Thermo Fisher Scientific, Inc.). The numbers of viral DNAs were normalized to the numbers of $\beta$-actin detected in the same samples (using forward 5'-CGGAACCGCTCATTGCC-3' and reverse 5'-ACCCAC ACTGTGCCCATCTA-3' primers (18). The reaction mixture for qPCR consisted of $4 \mu \mathrm{l}$ DNA extract, $12.5 \mu \mathrm{l}$ Power SYBR Green PCR master mix (Applied Biosystems; Thermo Fisher Scientific, Inc.), $0.5 \mu \mathrm{l}$ of each primer at $10 \mu \mathrm{M}$, and an ABI 7300 device (Applied Biosystems; Thermo Fisher Scientific, Inc.) was used. The amplification conditions were as follows: $95^{\circ} \mathrm{C}$ for $10 \mathrm{~min}$, followed by 40 cycles of $95^{\circ} \mathrm{C}$ for $15 \mathrm{sec}$ and $60^{\circ} \mathrm{C}$ for $1 \mathrm{~min}$.

A modified comparative $\mathrm{Cq}$ method $\left(2^{-\Delta \Delta \mathrm{Cq}}\right)$ was used for relative quantification of viral DNAs (19-21). The $\Delta$ Cq values $\left(\mathrm{Cq}_{\text {gene }}-\mathrm{Cq}_{\beta-\text {-Actin }}\right)$ were calculated, following which the $\Delta \Delta \mathrm{Cq}$ $\left(\Delta \mathrm{Cq}_{\text {treated }}-\Delta \mathrm{C \textrm {q } _ { \text { control } }}\right)$ were calculated. The relative numbers of

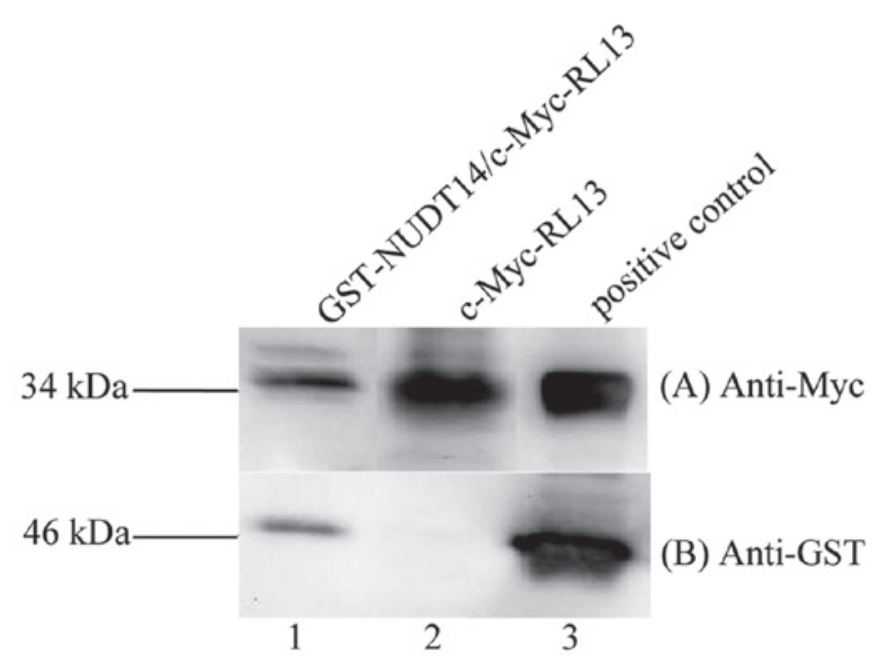

Figure 1. Interaction between host NUDT14 and viral RL13 proteins was analyzed using GST pull-down experiments. GST-tagged NUDT14 was used as a bait protein, and c-Myc-tagged RL13 was used as a prey protein. The recovered products were detected with either (A) mouse anti-c-Myc monoclonal antibody or (B) goat anti-GST monoclonal antibody. Lane 1, c-Myc-tagged RL13 and GST-labeled NUDT14 proteins captured by the MagneGST particles. Lane 2, c-Myc-labeled protein expressed from pGBKT7-RL13 in vitro. Lane 3, (A) commercial c-Myc-positive control protein or (B) protein lysates of BL21 (DE3) transfected with the GST-NUDT14-expressing plasmid (pGEX-4T2-NUDT14). GST, glutathione S-transferase; NUDT14, nucleoside diphosphate-linked moiety X (nudix)-type motif 14 .

HCMV DNAs were described using the equation $2^{-\Delta \Delta C q}$ (22). The experiments were repeated three times.

Statistical analysis. Statistical significance was analyzed using analysis of variance (ANOVA). $\mathrm{P}<0.05$ was considered to indicate a statistically significant difference. Data are presented as the means \pm standard deviation. All statistical analyses were computed using SPSS (Version 13.0; SPSS, Inc., Chicago, IL, USA), and graphs were produced using 


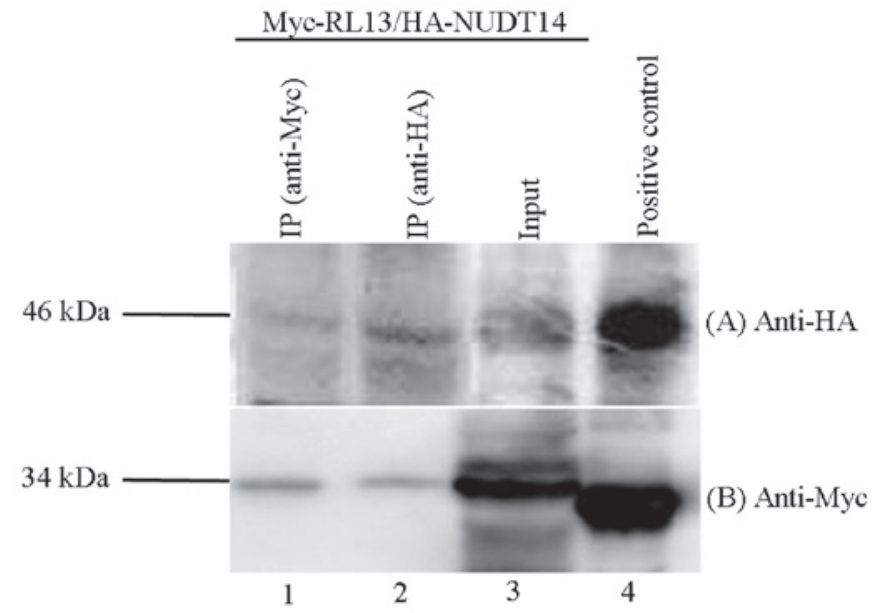

Figure 2. Interaction between c-Myc-labeled RL13 and HA-labled NUDT14 was identified by co-immunoprecipitation. The pCMV-Myc-RL13 and pCMV-HA-NUDT14 plasmids were transiently co-transfected into HEK293 cells. Cell lysates were extracted $48 \mathrm{~h}$ post-transfection. The protein samples (input, lane 3) and samples immunoprecipitated with either anti-Myc IP (anti-Myc) in lane 1 or anti-HA IP (anti-HA) in lane 2, antibodies were analyzed using Western blotting with antibodies against (A) HA-tagged and (B) Myc-tagged, respectively. The positive control (lane 4) shows the commercial (A) HA-positive or (B) c-Myc-positive control proteins. The c-Myc-labeled RL13 and HA-labeled NUDT14 proteins were determined in the recovered products immunoprecipitated with the antibodies of anti-c-Myc and anti-HA, respectively. IP, immunoprecipitation; HA, hemagglutinin; NUDT14, nucleoside diphosphate-linked moiety X (nudix)-type motif 14 .

GraphPad Prism 5 (GraphPad Software, Inc., San Diego, CA, USA).

\section{Results}

Identification of NUDT14 as a candidate binding protein of HCMV RL13 using yeast two-hybrid screening. The candidate, pACT2-NUDT14, was screened to examine its interaction with RL13. The transformation efficiency was almost $1.1 \times 10^{5} \mathrm{cfu} / \mu \mathrm{g}$, and 14 yeast colonies produced positive results. Sequencing and BLAST analysis indicated that 10 positive candidates interacted with the RL13 protein (Table I), one of which was $100 \%$ identical to that of the human NUDT14 sequence in the National Center for Biotechnology Information (Genbank no. NM177533.3).

Identification of the interaction between HCMV RL13 and host NUDT14 using a GST-pull-down assay. To further detect the binding between HCMV RL13 and host NUDT14 in vitro, GST-tagged NUDT14 was used as a bait protein and c-Myc-tagged RL13 was used as the prey protein in the GST-pull down assay. As shown in Fig. 1, a c-Myc-labeled RL13 protein ( $34 \mathrm{kDa})$ was captured, with a GST-tagged NUDT14 protein of $\sim 46 \mathrm{kDa}$ using MagneGST particles, but not with GST alone. These results showed that RL13 had the ability to interact with NUDT14 in vitro.

Determination of the interaction between HCMV RL13 and host NUDT14 by co-immunoprecipitation. To further confirm the interaction between RL13 and NUDT14, a co-IP assay was performed. As shown in Fig. 2, the c-Myc-labeled RL13 and HA-labeled NUDT14 proteins were assessed in the recovered products following immunoprecipitation with either anti-c-Myc or anti-HA antibodies (lanes 1 and 2, respectively). The input indicated that the protein levels of c-Myc-labeled RL13 and HA-labeled NUDT14 in the HEK293 cells were detected with anti-c-Myc and anti-HA antibodies, respectively (Fig. 2; lane 3). The positive control showed that the target proteins were correct in position and size (Fig. 2; lane 4). These results confirmed the interaction between the HCMV RL13 and host NUDT14 proteins in human cells.

RL13 and NUDT14 proteins are co-localized in human cells by fluorescence confocal microscopy. To determine whether the HCMV RL13 and NUDT14 proteins localized within the same cellular compartment, the HEK293 cells were transfected with pEGFP-RL13 (GFP-RL13), pDsRed-NUDT14 (RFP-NUDT14) or the two plasmids together, in the present study. The resulting merged image represented regions of overlap among the GFP-, RFP- and DAPI-stained images. The GFP-RL13 fusion proteins were localized predominantly in the HEK293 cell membrane and cytoplasm (Fig. 3A). Similarly, The RFP-NUDT14 fusion proteins were predominantly localized in the HEK293 cell membrane and cytoplasm (Fig. 3B). The fluorescent-labeled GPF-RL13 and RFP NUDT14 proteins were spatially co-localized and expressed simultaneously in the HEK293 cell membrane and cytoplasm (Fig. 3C). Furthermore, the results showed that the co-localization of the two proteins had no effects on the distribution of either individually in the uninfected cells.

Effect of NUDT14 protein on HCMV DNA replication. To investigate the effect of overexpressing NUDT14 on HCMV DNA replication, a stable NUDT14-expressing cell line, U373-S, was constructed. No differences were observed between the stable cell lines and the U373MG cells in growth characteristics or survival. In the stable U373-S cell lines, the protein expression of NUDT14 was five-fold higher than in the U373MG cells and the empty vector-transfected cells (U373-C; Fig. 4A).

To investigate the effect of underexpressing NUDT14 on HCMV DNA replication, the U373-S cells were transfected with NUDT14-specific siRNA molecules (S-siRNA). Compared to the U373-S cells, the underexpression of NUDT14 mediated by specific siRNA showed no effect on cell growth during the investigation. The results of the Western blotting results showed that the protein expression of NUDT14 in the S-siRNA-1- and S-siRNA-2-transfected cells collected $72 \mathrm{~h}$ post-HCMV infection were significantly reduced, by $84.14 \%$, compared with the C-siRNA-transfected cells (Fig. 4A).

To determine whether changes in the expression of NUDT14 altered HCMV DNA replication, viral DNA copies were detected using qPCR. At $72 \mathrm{~h}$ post-infection, no significant difference in the relative copy number of HCMV, namely UL83/ $\beta$-actin, in the U373-S cells stably expressing NUDT14, relative to those in the U373-C, U373MG and C-siRNA cells. However, following a decrease in the expression of NUDT14, there was a 20 -fold increase in the number of HCMV copies in the infected cells treated with the two NUDT14-specific $\mathrm{S}$-siRNA-1 (ANOVA $\mathrm{P}=0.036$ ) and $\mathrm{S}$-siRNA-2 (ANOVA $\mathrm{P}=0.002$ ), compared with those in the cells transfected with 
A

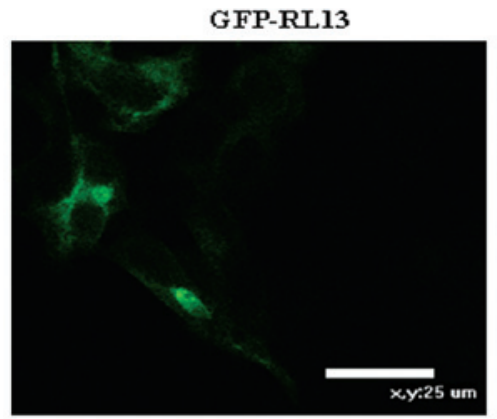

B

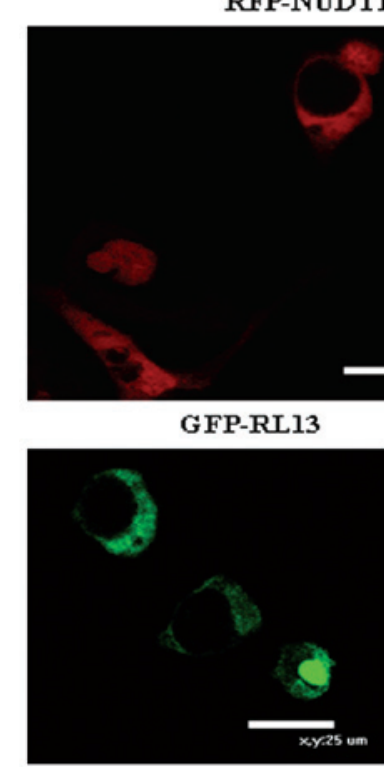

DAPI

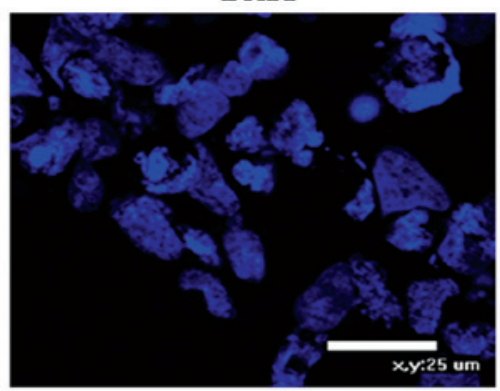

DAPI

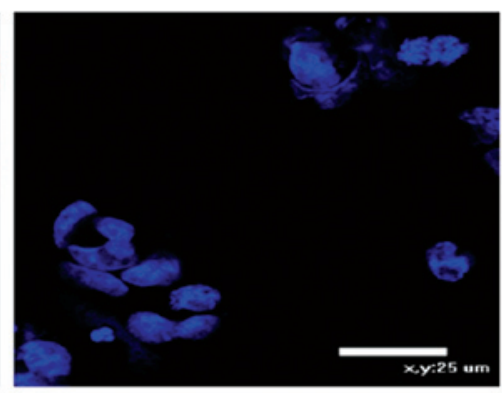

DAPI

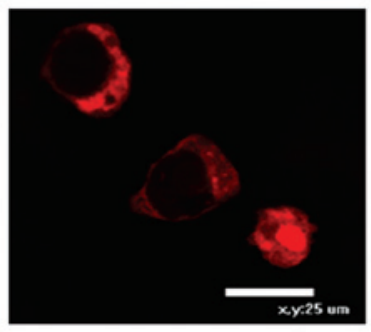

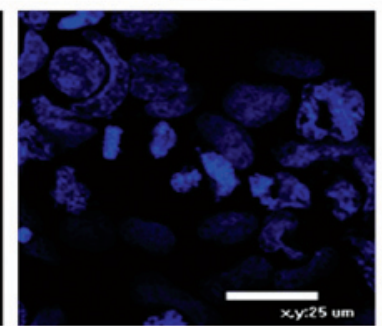

Merge

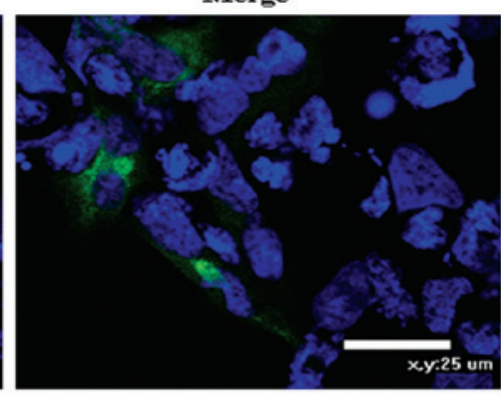

Merge

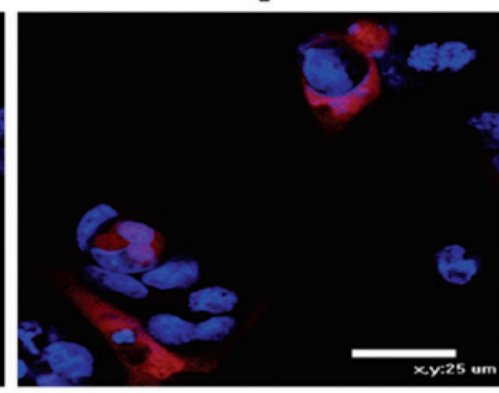

Merge

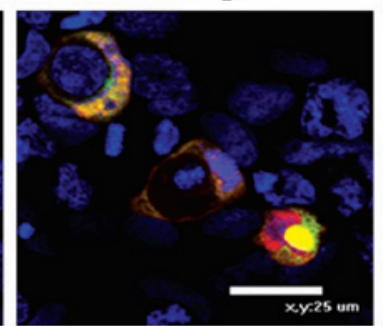

Figure 3. Localization of RL13 and NUDT14 proteins expressed in HEK293 cells. HEK293 cells were cultured in Dulbecco's modified Eagle's medium with $10 \%$ fetal calf serum. At 75\% confluence, cells were transiently transfected with (A) EGFP-RL13, (B) RFP-NUDT14 or (C) both using Lipofectamine 2000. At $48 \mathrm{~h}$ post-transfection, DAPI (blue) was used for staining the nuclear DNA. The distribution of the expressed proteins was detected under a fluorescent confocal microscope. 'Merge' represents the overlapping image of fluorescence from the expressed proteins and nuclear staining in the same field of view. NUDT14, nucleoside diphosphate-linked moiety X (nudix)-type motif 14; RFP, red fluorescent protein; GFP, green fluorescent protein.

A

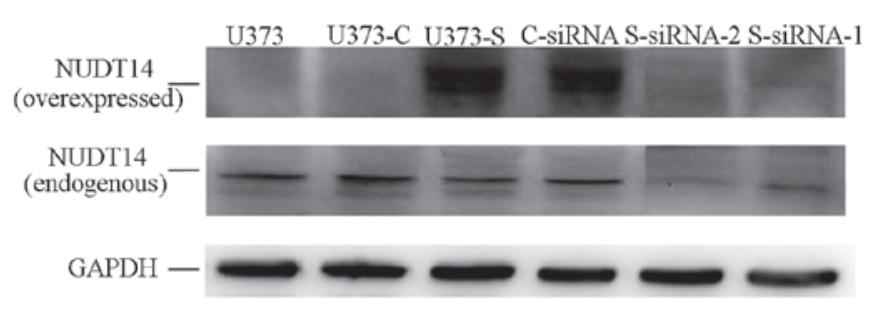

B

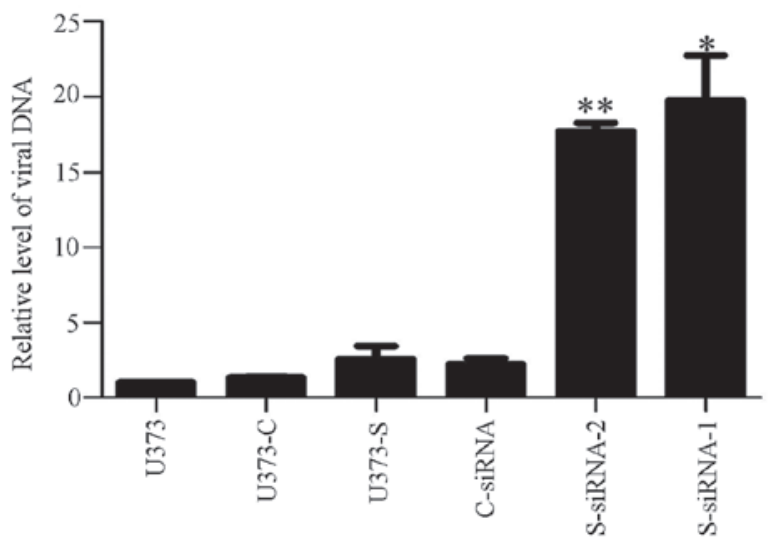

Figure 4. Detection of NUDT14 stable cell lines and the effects of overexpression and underexpression of NUDT14 on viral replication. (A) Protein levels of NUDT14 in the cells were assessed using Western blot analysis using rabbit anti-NUDT14 antibody. The expression of cellular GAPDH was determined as the internal control. (B) Viral DNA copies were detected using reverse transcription-quantitative polymerase chain reaction analysis. The relative levels of viral DNA in different cells are expressed as the mean \pm standard deviation of three independent repeated experiments, and determined by the number of viral DNA copies in the cells, compared with the number in the U373MG cells. ${ }^{*} \mathrm{P}<0.05$ and ${ }^{* *} \mathrm{P}<0.01$ vs. cells transfected with C-siRNA. NUDT14, nucleoside diphosphate-linked moiety X (nudix)-type motif 14; GAPDH, glyceraldehyde-3-phosphate dehydrogenase; siRNA, small interfering RNA; C-siRNA, control siRNA; S-siRNA, specific siRNA.

C-siRNA and the U373-S cells. The fact that the S-siRNA-1 and S-siRNA-2 molecules exhibited the same phenotype indicated that there was no off-target of the siRNAs in the RNA interference experiments (Fig. 4B). These results suggested that 
inhibiting the expression of NUDT14 in the HCMV-infected cells increased viral replication.

\section{Discussion}

Replication of herpes virus DNA is regarded as a complex and controlled event during lytic infection $(23,24)$. Understanding the interaction between the virus and human host is pivotal for clarifying the viral replication mechanism. Stanton et al indicated that RL13 exerted independent, suppressive effects on HCMV growth in fibroblasts, as well as epithelial cells $(10,11)$. However, the mechanism remains to be elucidated.

In the present study, in order to investigate novel human proteins, which potentially interact with the HCMV RL13 protein for elucidating the viral replication mechanism, a yeast two-hybrid screen was performed. The results showed that RL13 interacted with 10 candidates, one of which was human NUDT14 (Table I). As a member of the Nudix hydrolase family, NUDT14 encodes a UDP-glucose pyrophosphatase (UGPPase), which reversibly catalyzes the formation of UDP-glucose and pyrophosphate from UTP and glucose 1-phoshpate in the presence of $\mathrm{Mg}^{2+}$ (25). To further identify the potential interaction, a GST pull-down experiment was performed. As shown in Fig. 1, c-Myc-RL13 was specifically bound to GST-NUDT14 in vitro. To examine the interaction between the RL13 protein and NUDT14 protein in more detail, a co-immunoprecipitation experiment using HEK293 cells was performed. As shown in Fig. 2, c-Myc-RL13 was specifically immunoprecipitated with HA-NUDT14. To further characterize the interaction, co-localization of these two proteins was confirmed by confocal microscopy analysis. As shown in Fig. 3, GFP-RL13 and RFP-NUDT14 were spatially co-localized in the HEK293 cells. These results provided precise evidence that the RL13 protein interacted with the NUDT14 protein in mammalian cells. The present study is the first, to the best of our knowledge, to report on the interactions between any HCMV proteins and NUDT14 proteins.

NUDT14 encodes a UGPPase, which belongs to the Nudix hydrolases superfamily with versatile, $\mathrm{Mg}^{2+}$-requiring, 'housecleaning' characteristics, and may exert housecleaning functions in eliminating toxic metabolites $(26,27)$. It has been reported that UGPPase may function in modulating glycogen metabolism, and may be vital in connecting the gluconeogenic process with other metabolic pathways to meet the physiological and biochemical requirements of the mammalian cell (28-31). The results of the present study suggested s specific interaction between NUDT14 and RL13 in the cell membrane and cytoplasm, and further investigations may determine whether this interaction affects HCMV DNA replication, for the development of antiviral strategies to prevent HCMV infection and disease. In reference to the above results, the present study investigated whether the RL13 and NUDT14 proteins are involved in viral replication.

In the present study, the underexpression of NUDT14 by a specific siRNA in HCMV-infected cells resulted in an increase in the level of viral DNA (Fig. 4). UGPPase may exert a housecleaning role in eliminating toxic metabolites, as a member of the nudix hydrolases $(26,27)$. As a specific type of toxic metabolite, HCMV infection may be eliminated by cellular
UGPPase. The underexpression of NUDT14 by specific siRNA in HCMV-infected cells may impair housecleaning by NUDT14, which may benefit viral DNA replication.

The present study provided additional evidence that the overexpression of NUDT14 had no significant effects on host cell growth or viral DNA replication in the U373-S cell line. This may be due to the level of endogenous NUDT14 being sufficient to maintain the normal physiological function of cells. This hypothesis requires further experimental verification, as only the interaction between HCMV RL13 and host NUDT14, and the effects of altering the expression of NUDT14 in U373MG cells on viral DNA replication, were confirmed in the present study. Whether changes in the expression of NUDT14 in other HCMV-permissive cells affects viral DNA replication remains to be elucidated.

In conclusion, the present study identified RL13 as a late gene in HCMV lytic infection, shown by its interaction with the host protein, NUDT14, and that inhibition of the expression of cellular NUDT14 increased HCMV DNA replication. These findings assist in beginning to understand the mechanisms of the interaction between the HCMV RL13 protein and the NUDT14 host factor during infection. An improved understanding of the interaction between HCMV RL13 and host proteins may provide insight into the fundamental cellular pathways involved in viral binding and entry in lytic infections.

\section{Acknowledgements}

This study was jointly sponsored by a grant from the National Natural Science Foundation of China (grant nos. 81171580 and 81171581) and the Outstanding Scientific Fund of Shengjing Hospital (grant no. 201105).

\section{References}

1. Bissinger AL, Sinzger C, Kaiserling E and Jahn G: Human cytomegalovirus as a direct pathogen: Correlation of multiorgan involvement and cell distribution with clinical and pathological findings in a case of congenital inclusion disease. J Med Virol 67: 200-206, 2002.

2. Plachter B, Sinzger C and Jahn G: Cell types involved in replication and distribution of human cytomegalovirus. Adv Virus Res 46: 195-261, 1996.

3. Sinzger C, Grefte A, Plachter B, Gouw AS, The TH and Jahn G: Fibroblasts, epithelial cells, endothelial cells and smooth muscle cells are major targets of human cytomegalovirus infection in lung and gastrointestinal tissues. J Gen Virol 76: 741-750, 1995.

4. Mocarski ES: Cytomegaloviruses. In: Fields Virology. Fields BN, Knipe DM and Howley PM (eds.) Lippincott-Raven, Philadelphia, Pennsylvania, USA, pp2447-pp2492, 2006.

5. Sinzger C, Plachter B, Grefte A, The TH and Jahn G: Tissue macrophages are infected by human cytomegalovirus in vivo. J Infect Dis 173: 240-245, 1996.

6. Kahl M, Siegel-Axel D, Stenglein S, Jahn G and Sinzger C: Efficient lytic infection of human arterial endothelial cells by human cytomegalovirus strains. J Virol 74: 7628-7635, 2000.

7. Riegler S, Hebart H, Einsele H, Brossart P, Jahn G and Sinzger C: Monocyte-derived dendritic cells are permissive to the complete replicative cycle of human cytomegalovirus. J Gen Virol 81: 393-399, 2000.

8. Dolan A, Cunningham C, Hector RD, Hassan-Walker AF, Lee L, Addison C, Dargan DJ, McGeoch DJ, Gatherer D, Emery VC, et al: Genetic content of wild-type human cytomegalovirus. J Gen Virol 85: 1301-1312, 2004.

9. Li M, Ma Y, Ji Y, He R, Qi Y, Sun Z, Wang N, Gao S and Ruan Q: Human cytomegalovirus RL13 gene transcripts in a clinical strain. Virus Genes 43: 327-334, 2011. 
10. Stanton RJ, Baluchova K, Dargan DJ, Cunningham C, Sheehy O, Seirafian S, McSharry BP, Neale ML, Davies JA, Tomasec P, et al: Reconstruction of the complete human cytomegalovirus genome in a BAC reveals RL13 to be a potent inhibitor of replication. J Clin Invest 120: 3191-3208, 2010.

11. Dargan DJ, Douglas E, Cunningham C, Jamieson F, Stanton RJ, Baluchova K, McSharry BP, Tomasec P, Emery VC, Percivalle E, et al: Sequential mutations associated with adaptation of human cytomegalovirus to growth in cell culture. J Gen Virol 91: 1535-1546, 2010.

12. Cortese M, Calò S, D'Aurizio R, Lilja A, Pacchiani N and Merola M: Recombinant human cytomegalovirus (HCMV) RL13 binds human immunoglobulin G Fc. PLoS One 7: e50166, 2012.

13. To A, Bai Y, Shen A, Gong H, Umamoto S, Lu S and Liu F: Yeast two hybrid analyses reveal novel binary interactions between human cytomegalovirus-encoded virion proteins. PLoS One 6: e17796, 2011

14. McMahon TP and Anders DG: Interactions between human cytomegalovirus helicase-primase proteins. Virus Res $86: 39-52$, 2002.

15. Stepanchenko NS, Novikova GV and Moshkov IE: Protein quantification. Russ J Plant Physiol 58: 727-742, 2011.

16. Liu F and Altman S: Inhibition of viral gene expression by the catalytic RNA subunit of RNase P from Escherichia coli. Genes Dev 9: 471-480, 1995.

17. Miller AD and Rosman GJ: Improved retroviral vectors for gene transfer and expression. Biotechniques 7: 980-982, 984-986, 989-990, 1989.

18. Hänfler J, Kreuzer Ka, Laurisch K, Rayes N, Neuhaus P, Schmidt CA and Oettle H: Quantitation of cytomegalovirus (hCMV) DNA and beta-actin DNA by duplex real-time fluorescence PCR in solid organ (liver) transplant recipients. Med Microbiol Immunol 192: 197-204, 2003.

19. Su J, Zhu Z, Xiong F and Wang Y: Hybrid cytomegalovirus-U6 promoter-based plasmid vectors improve efficiency of RNA interference in zebrafish. Mar Biotechnol (NY) 10: 511-517, 2008.

20. Cahill AL, Moore JM, Sabar FI and Harkins AB: Variability in RNA interference in neuroendocrine PC12 cell lines stably transfected with an shRNA plasmid. J Neurosci Methods 166: 236-240, 2007.
21. Livak KJ and Schmittgen TD: Analysis of relative gene expression data using real-time quantitative PCR and the 2(-Delta Delta C(T)) Method. Methods 25: 402-408, 2001.

22. Duan QJ, Tao R, Hu MF and Shang SQ: Efficient inhibition of human cytomegalovirus UL122 gene expression in cell by small interfering RNAs. J Basic Microbiol 49: 531-537, 2009.

23. Mocarski ES, Shenk T and Pass RF: Cytomegalovirus. In: Fields virology. Knipe DM, Howley PM, Griffin DE, et al: (eds.) Lippincott Williams \& Wilkins, Philadelphia, PA, pp2701-2772, 2007.

24. Roizman B, Knipe D and Whitley R: Herpes simplex viruses. In: Fields virology. Knipe DM and Howley PM (eds.) Lippincott Williams \& Wilkins, Philadelphia, Pennsylvania, USA, pp2503-pp2601, 2007.

25. Aksamit RR and Ebner KE: Purification, properties and kinetic analysis of UDP-glucose pyrophosphorylase from bovine mammary tissue. Biochim Biophys Acta 268: 102-112, 1972.

26. Bessman MJ, Frick DN and O'Handley SF: The MutT proteins or 'Nudix' hydrolases, a family of versatile, widely distributed, 'housecleaning' enzymes. J Biol Chem 271: 25059-25062, 1996.

27. McLennan AG: The nudix hydrolase superfamily. Cell Mol Life Sci 63: 123-143, 2006.

28. Heyen CA, Tagliabracci VS, Zhai L and Roach PJ: Characterization of mouse UDP-glucose pyrophosphatase, a Nudix hydrolase encoded by the Nudt14 gene. Biochem Biophys Res Commun 390: 1414-1418, 2009.

29. Yagi T, Baroja-Fernández E, Yamamoto R, Muñoz FJ, Akazawa T, Hong KS and Pozueta-Romero J: Cloning, expression and characterization of a mammalian Nudix hydrolase-like enzyme that cleaves the pyrophosphate bond of UDP-glucose. Biochem J 370: 409-415, 2003.

30. Rodriguez-López M, Baroja-Fernández E, Zandueta-Criado A and Pozueta-Romero J: Adenosine diphosphate glucose pyrophosphatase: A plastidial phosphodiesterase that prevents starch biosynthesis. Proc Natl Acad Sci USA 97: 8705-8710, 2000.

31. Moreno-Bruna B, Baroja-Fernández E, Muñoz FJ, Bastarrica-Berasategui A, Zandueta-Criado A, Rodriguez-Ló pez M, Lasa I, Akazawa T and Pozueta-Romero J: Adenosine diphosphate sugar pyrophosphatase prevents glycogen biosynthesis in Escherichia coli. Proc Natl Acad Sci USA 98: 8128-8132, 2001. 